Hydrology and Earth System Sciences, 8(6), 1221-1223 (2003) C EGU

\title{
Author Index to Volume 8
}

Acreman, M.C. 673,861

Adamson, P.T. 1118

Aigang Lu 115

Akyürek, Z. 1200

Alam, M.S. 392

Anctil, F. 940

Andersen, H.-E. 651

Archer, D.R. 47

Aschan, G. 1065

Aspden, R.J. 160

Baborowski, M. 135

Bari, M. 903

Bastrup-Birk, A. 764

Bates, R. 160

Bayer, A. 2

Bégassat, P. 8

Bergonzini, L. 256

Bernal, S. 729

Beven, K. 88

Blyth, E.M. 923

Bottero, J.-Y. 8

Bouten, W. 751

Bradley, C. 1164

Branchu, P. 256

Brandt, C. 345

Bridcut, E.E. 366

Briers, R.A. 545

Brittain, S.A. 436

Broadmeadow, S. 286, 355

Brown, C.M. 1179

Bunch, M.A. 122

Burke, E.J. 1179

Butterfield, D. 695, 706, 846

Butterini, A. 729

Buytaert, W. 108

Carey, S.K. 877

Catarino, J.B. 151

Clay, A. 1164

Collen, P. 567

Coward, P. 377
Cowpertwait, P.S.P. 993

Cresser, M.S. 355, 392

Crossley, A. 377

Dabrowski, M. 79

Dahl, M. 1153

Dai, S.B. 1210

Darling, W.G.

Davidson, I.R. 160

Davies, H. 823

Davies, T.D. 409

De Bièvre, B. 108

De Groote, J. 191, 205

de Jong, C. 1065

De Smedt, F. 1129

de Vries, W. 813

Deckers, J. 108

Deelstra, J. 651

Deschamps, P. 35

Diodato, N. 103

Dise, N. 597, 686

Dobson, M. 550, 560

Donaghy, M.J. 449

Douselance, R. 35

Duck, R.W. 151

Dunbar, M.J. 861

Dunn, S.M. 205

Durand, P. 793

Essery, R. 1045

Farmer, R.A. 279

Fernando, R.C. 235

Ferrier, R.C.

Feyen, J. 931

Figueiredo da Silva, J. 151

Finch, J. 345, 923

Fisher, J. 673

Flynn, N.J. 834

Forkel, R. 1030

Fowler, H.J. 47

Fox, N.I. 1001
Franks, S.W. 1118

Fraser, A. 88

Friese, K. 135

Futter, M.N. 846

Gao, A. 1210

García-Santos,, G. 1065

Gebremeskel, S. 1129

Gee, J.H.R. 545

Gerrard, A.J. 1164

Ghaleb, B. 35

Giller, P. 314

Goeller, N.T. 877

Granlund, K. 717

Grant, S.J. 327

Gundersen, P. 764

Guo, S. 247

Hall, J. 355

Hall, M.J. 235, 1174

Halliwell, D. 88

Hannah, D.M. 449

Haria, A.H. 334

Harlow, R.C. 1179

Harriman, R. 366

Harrod, R. 88

Harvey, F.J. 377

Haygarth, P. 88

Heathwaite, A.L. 834

Heuvelmans, G. 931

Hewett, C.J.M. 834

Hewitt 601, 614

Hill, L. 485, 503, 601, 614

Hill, T.J. 533

Hillaire-Marcel, C. 35

Hirst, D. 422

Hoffmann, L. 1129

Holmes, M.G.R. 891

Hongxi Pand, 115

Hornung, M. 355

Hudson, J.A. 327

Hughes, S. 436 
Ingleby, K. 377

Iroumé, A. 23

Jansons, V. 651

Jarvie, H.P. 614

Jarvis, S. 88

Jones, D.A. 183, 973

Jones, M.C. 578

Jones, M.E. 578

Juan Gu 115

Kalma, J.D. 1217

Kansakar, S.R. 891

Karvonen, T. 706

Kaste, Ø. 695. 778

Keay, E.J. 567

Keim, R.F. 23

Kennedy, M.P. 663

Kjeldsen, T.R. 183

Knoche, R. 1030

Konicek, A. 1186

Kros, J. 813

Krug, E.C. 98

Kuczera, G. 1118

Kunstmann, H. 1030

Kyllmar, K. 651

Laize, C.L.R. 1103

Lambert, M.F. 1118

Langan, S.J. 277, 355, 392, 422, 589

Langusch, J. 742

Lauzon, N. 940

Leng, M.J. 1164

Lepiller, M. 1051

Lepistö, A. 717, 778

Li, P. 2010

Lilly, A. 191, 205

Link, T.E. 23

Lischeid, G. 742

Liu, Y.B. 1129

Loigu, E. 651

Machefert, S.E. 686

Machín, J. 1193

Mackay, R. 122

Malcolm, I.A. 449

Mangin, L. 1051

Marszelwski, W. 79

Marzol, M.V. 1065

Mathevet, T. 1051

McCartney, M.P. 266
McCulloch, J.S.G. 119

McGechan, M. 191

McNish, J. 366

Metcalfe, A.V. 1118

Michelot, J.-L. 35

Miklanek, P. 1186

Mols-Dijkstra, J.P. 813

Morrison, B.R.S. 567

Murphy, K.J. 663

Muys, B. 931

Mynett, A.R. 235

Nash, D. 88

Naudet, V. 8

Navas, A. 1193

Neal, C. 277, 409, 460, 485, 503, 521, $533,599,614,823,846,901$

Neal, M. 460, 503, 485, 601, 614

Négrel, P. 959

Ness, L. 409

Nisbet, T.R. 277, 279, 286, 589

Norris, D.A. 436

O’Connor, K.M. 247

O'Halloran, J. 314

Ormerod, S.J. 277, 578, 589

Ott, B. 62

Page, T. 88

Pegram, G.G.S. 220

Pekar, J. 1186

Pekarova, P. 1186

Perkins, R.G. 160

Pers, B.C. 1153

Petelet-Giraud, E. 959

Peterson, D.M. 160

Petti, J.R. 1179

Pfister, L. 1129

Phillips, D.R. 578

Pretty, J.L. 550, 560

Quinn, P.F. 834

Quinton, W.L. 877

Raat, K.J. 751

Rankinen, K. 695, 706, 717, 778

Ranzi, R.

Rees, H.G. 891

Reeves, A. 121

Rekolainen, S. 651

Revil, A. 8
Reynolds, B. 306, 355, 409, 436, 460, 485,503

Riera, J.L. 729

Rizzo, E. 8

Roberts, J. 277, 589, 1218

Robinson, M. 327, 345, 923

Rogora, M.

Rosbjerg, D. 1090

Rosier, P. 923

Rosso, R.

Roth, K. 2

Sabater, F. 729

Schneider, K. 1030

Schrøder, T.M. 1090

Schulz, O. 1076

Scott, R.L. 1179

Seibert, J. 1

Shand, P. 334

Sheppard, L.J. 377

Shi, Z. 2010

Shuttleworth, W.J. 1179

Skaugset. A.E. 23

Skeffington, R. 601

Skiba, U. 377

Skowron, R. 79

Smart, R.P. 392

Smettem, K.R.J. 903

Snook, D.L. 636

Soulsby, C. 449

Stålnacke, P. 651

Stevens, P.S. 436

Stratford, C.J. 266

Stutter, M.I. 392

Sutton, M.A. 191

Svensson, C. 973

Tellam, J.H. 122

Tietema, A. 751, 803, 813

Tombul, M. 1200

Tumas, R. 651

Turner, B.L. 88

Turner, P. 122

Uhlenbrook, S. 62

Ünal Sorman, A. 1200

Vagstad, N. 651

Valero Garcés, B. 1193

van den Boogaard, H.F.P. 235, 1174

Vardy, S. 160 
Vázquez, E. 729

Vinten, A.J.A. 191, 205

Viviroli, D. 1016

Vogel, H.-J. 2

von Tümpling, jr., W. 135

Vrugt, J.A. 751

Wade, A.J. 599, 614, 846

Weingartner, R. 1016

Werner, M.G.F. 1141

Wesson, S.M. 220

Whitehead, P.G. 533, 636, 834

Whiting, J.P. 1118

Wickham, H. 485, 503, 601, 614

Widery, D. 959

Williams, B. 460, 485, 503

Williams, R.J. 266

Winstanley, D. 98

Woodin, S.J. 392

Woods, C. 436

Wyatt, R. 601, 614

Wyseure, G. 108

Xiong, L. 247

Yang, S.L. 170, 1210

Young, A.R. 891

Youngson, A.F. 449

Yuanqing He, 115

Zhang, J. 170

Zhao, H.Y. 1210

Zhonglin Zhang, 115

Zhu, J. 170 